\title{
Utilização de uma bomba de infusão contínua como geradora de vácuo para a aspiração folicular transvaginal guiada pela ultra-sonografia
}

Use of a permanent infusion pump as vacuum generator for ovum pick-up

\section{Utilización de una bomba de infusión continua como generadora de vacuo para la aspiración folicular transvaginal guiada por la ultrasonografía.}

\section{Marcelo Marcondes Seneda; ${ }^{1}$ Karina Cristina Puggesi Rubin; ${ }^{2}$ Wanessa Blaschi; ${ }^{3}$ Lívia Aires Lisboa; ${ }^{4}$ José Henrique Fortes Pontes ${ }^{5}$}

Departamento de Clínicas Veterinárias da Universidade Estadual de Londrina (UEL). Londrina, PR, Brasil

\section{Resumo}

Divulgar um equipamento alternativo para a geração de vácuo, capaz de permitir a recuperação de oócitos em quantidade e qualidade compatíveis com bombas feitas especificamente para este fim. Utilizou-se uma bomba de infusão contínua, cuja aplicabilidade para fins de aspiração foi possível graças ao modo inverso de utilização do equipo de infusão. A extremidade destinada ao frasco de infusão foi acoplada no tubo de aspiração folicular, enquanto a extremidade do equipo destinada ao cateter do paciente foi mantida livre. Obtiveram-se resultados similares aos encontrados em equipamentos designados especificamente para aspiração folicular. A bomba de infusão contínua pode ser uma alternativa viável para a obtenção in vivo de oócitos bovinos.

Palavras-chave: Folículo ovariano. Oócitos. Aspiração folicular. Vácuo. Ultra-sonografia. Bovinos.

${ }^{1}$ Professor Doutor. Curso de Medicina Veterinária da UEL. CRMV-PR 5145

${ }^{2}$ Mestranda em Ciência Animal. Curso de Medicina Veterinária da UEL. CRMV-PR 6712

${ }^{3}$ Mestranda em Ciência Animal. Curso de Medicina Veterinária da UEL. CRMV-SP 14. 375

${ }^{4}$ Graduanda do 52 ano do Curso de Medicina Veterinária da UEL

${ }^{5}$ Médico Veterinário. Diretor Técnico - In Vitro Brasil. CRMV-SP 07298 


\section{Introdução}

Da grande população de folículos primordiais presentes nos ovários das fêmeas bovinas por ocasião do nascimento, apenas uma fração mínima chega a se desenvolver até o estágio de folículo préovulatório; a maioria, cerca de $99,9 \%^{1}$ sofre atresia após iniciar o crescimento in vivo. ${ }^{2}$ A efetiva exploração dessa reserva nas fêmeas tem sido um desafio para técnicos e pesquisadores em todo o mundo. Dessa forma, a produção in vitro de embriões (PIVE) demonstra ser uma boa alternativa para a utilização dessa reserva presente nos ovários das fêmeas bovinas. A PIVE já vem sendo utilizada em vários países, mas é no Brasil que se verifica um número surpreendente de embriões gerados a partir desse método, sendo o país líder mundial em produzidos nascidos por esse método. Dentre os principais aspectos relacionados a esta expressiva disseminação da técnica, pode-se apontar o domínio da PIVE por laboratórios privados. Originalmente, todo o processo de produção de embriões in vitro era restrito às equipes das empresas dedicadas a essa atividade. Atualmente, verifica-se em processo crescente a terceirização de algumas etapas, principalmente a obtenção in vivo dos oócitos. A aspiração folicular transvaginal apresenta-se como mais uma opção de serviço a ser prestado pelos profissionais da área de reprodução aplicada. $\mathrm{O}$ alto custo dos equipamentos necessários à aspiração folicular apresenta-se como fator de restrição para aqueles profissionais.

Neste contexto, a proposta do presente trabalho é apresentar um equipamento alternativo para geração de vácuo, visando permitir acesso mais amplo aos interessados em atuar no processo de obtenção in vivo de oócitos para a indústria de tecnologia de embriões.

\section{Revisão de Literatura}

A primeira etapa da PIVE é a obtenção in vivo dos oócitos, que ocorre com a técnica de aspiração folicular transvaginal, ou ovum pick-up (OPU). Há mais de uma década a OPU tem sido a melhor opção para a recuperação de oócitos in vivo na espécie bovina. ${ }^{3}$
Objetivando aumentar a eficiência e reduzir o investimento inicial para realização da técnica, a OPU tem sido estudada por diversos grupos. ${ }^{4}$ Segundo Bols $^{5}$ (1997), os principais fatores a serem analisados reúnem-se nos aspectos biológicos e nos de ordem técnica. Os aspectos biológicos constituem um conjunto de variáveis difíceis de serem modificadas, pois compreendem a condição fisiológica da doadora, principalmente raça, idade, escore corporal, atividade ovariana e a própria variação individual.

Os aspectos técnicos compreendem tipo e frequiência do transdutor; comprimento, diâmetro e bisel da agulha de punção e a pressão de vácuo. $\mathrm{O}$ conhecimento e a melhoria destas variáveis técnicas podem contribuir para uma possível redução de custos, abrindo a perspectiva de veterinários autônomos realizarem a aspiração folicular, para posterior envio dos oócitos às centrais de PIVE.

Segundo Hashimoto et al. ${ }^{6}$ (1999), a freqüência do transdutor constitui-se em variável importante no processo de recuperação dos oócitos. Há citações de freqüências entre $3.5 \mathrm{MHz} ;{ }^{7} 5.0 \mathrm{MHz} ;{ }^{3,8.9} 6.5 \mathrm{MHz}^{10,11}$ e $7.5 \mathrm{MHz} .{ }^{12,13}$ Pela proximidade do ovário ao transdutor no momento da punção, admitem-se freqüências entre 5 e $8 \mathrm{MHz}$ como adequadas para a realização da técnica. Além da freqüência, outra variável importante é o tipo de transdutor. O transdutor endovaginal humano, ou microconvexo, mostrou-se altamente eficiente às condições anatômicas de vacas pequenas e novilhas pré-púberes. ${ }^{14}$ Permitiu ainda ótima visualização dos folículos em vacas de grande porte, por facilitar a manipulação ovariana, apresentar uma imagem com alta resolução e possuir imagem com grande ângulo de abertura. ${ }^{15}$ Outra opção de transdutor é o transdutor linear (Figura 1). Apesar de os trabalhos iniciais mostrarem eficiência similar ao transdutor convexo ou setorial -, os mais utilizados para aspiração folicular transvaginal ${ }^{16-20}$ - a continuidade dos procedimentos demonstrou queda de eficiência de $10 \%$ a $20 \%$ para o transdutor linear, variando conforme a habilidade do operador. No entanto, o transdutor linear é amplamente difundido no segmento de reprodução assistida, e sua versatilidade deve ser considerada para a obtenção de oócitos in vivo, especialmente quando a finalidade do ultra-som envolver outras atividades além da aspiração folicular. 


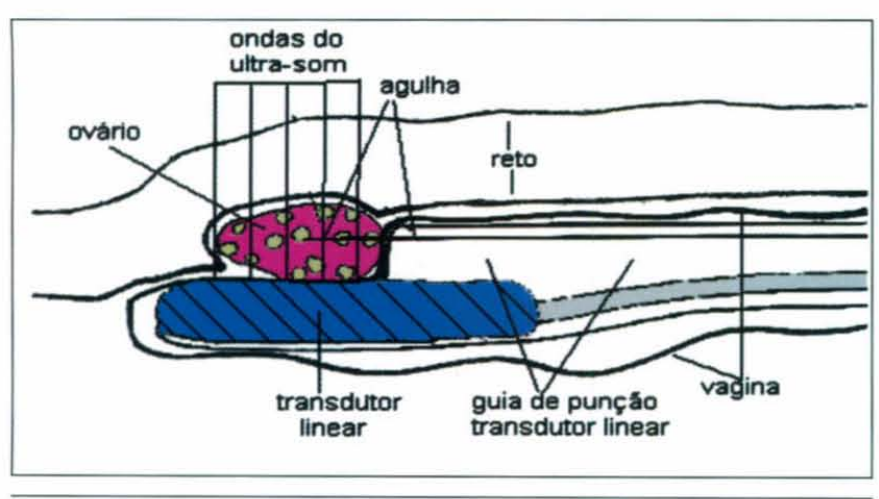

Figura 1 - Ilustração do posicionamento do transdutor linear para aspiração folicular transvaginal. (Fonte: adaptado de SENEDA et al., ${ }^{21}$ 2003)

Com relação às agulhas para aspiração, em princípio, utilizavam-se apenas as agulhas longas (55 $\mathrm{cm}$ ), produzidas especificamente para aspiração folicular. ${ }^{22}$ Essas agulhas mostraram-se altamente eficazes se utilizadas em um único animal. No entanto, devido ao seu alto custo, é comum que uma mesma agulha seja utilizada para vários animais, com possibilidade de danos ao estroma ovariano e pior recuperação de oócitos. Dessa forma, propôs-se a substituição por agulhas hipodérmicas descartáveis, atualmente uma tendência irreversível para a aspiração folicular. Bols et al. ${ }^{23}$ (1997) demonstraram a via-bilidade da utilização de agulhas hipodérmicas descartáveis de 18 e 19 Gauge (G) e $50 \mathrm{~mm}$ de comprimento. ${ }^{3}$ Estas, por sua vez, possuem restrita distribuição no mercado, mas são perfeitamente substituíveis pelas de $40 \mathrm{~mm}$. Portanto, as opções mais viáveis no mercado nacional seriam as agulhas $40 \times 8 \mathrm{~mm}, 40 \times 9 \mathrm{~mm}$ e 40 x $10 \mathrm{~mm}$. Considerando seu preço irrisório, torna-se possível utilizar uma agulha por vaca, ou mesmo uma por ovário, em animais com maior número de folículos. Assim, o procedimento de recuperação dos oócitos pode ser realizado com mínimos danos ao estroma ovariano.

Estreitamente relacionada com a agulha utilizada é a pressão de vácuo. ${ }^{24,25}$ Baixas pressões de vácuo, como $50 \mathrm{~mm} \mathrm{Hg}$, foram pouco eficientes para a aspiração, enquanto pressões maiores, como $120 \mathrm{~mm} \mathrm{Hg}$, danificaram o revestimento cumulus oophorus. Há uma grande variação entre os trabalhos, com valores de 40 a $400 \mathrm{~mm} \mathrm{Hg} .{ }^{18}$ Porém, esse dado deve ser considerado com reservas, já que todo o sistema - incluindo o comprimento e diâmetro de conexões, altura do equipamento de vácuo e diâmetro da agulha - pode influenciar na pressão de vácuo final. Para quantificar a pressão negativa de forma mais real, sugere-se mensurar o vácuo em volume de fluido por minuto. Mesmo assim, há variações consideráveis, de 4,4 a $40 \mathrm{ml}$ de água/minuto. ${ }^{11,22,25,26}$ $\mathrm{O}$ intervalo entre 10 a $20 \mathrm{ml}$ por minuto tem sido o mais utilizado atualmente, sendo pertinente a aferição a cada procedimento.

\section{Equipamentos utilizados na geração de vácuo para obtenção in vivo de oócitos}

As bombas de vácuo Handle Cook ${ }^{\circledR}$ foram especialmente designadas para obtenção de oócitos em vacas. ${ }^{22}$ Tais equipamentos possuem grande estabilidade para gerar vácuo, permitindo considerável eficiência na recuperação dos oócitos, tanto na quantidade como na capacidade de minimizar danos aos oócitos durante o procedimento. Além de nível mínimo de ruído, essa bomba apresenta recurso de vácuo máximo, bastante útil quando ocorre formação de coágulos no sistema de aspiração. Um aspecto desfavorável é seu custo elevado, tornandoa restrita às centrais de PIVE, fator que motivou a busca alternativa de bombas de vácuo.

Outra bomba específica para aspiração folicular presente no mercado é a Rocket Medical ${ }^{\circledR}$. Por um lado, possui grande estabilidade na pressão de vácuo e apresenta recurso de vácuo máximo, além de possuir nível de ruído moderado. Por outro, o investimento necessário para sua aquisição também é considerável.

Em busca de alternativas de baixo custo, verificou-se a utilização de bombas de vácuo de uso hospitalar, destinadas à aspiração de fluidos e secreções. Constituem alternativa válida, devido ao seu baixo custo e facilidade de aquisição. No entanto, bombas desse tipo apresentam instabilidade na manutenção do vácuo e dificuldade para o ajuste da vazão desejada, além de elevado nível de ruído. 
Uma opção interessante apresentada neste artigo é a bomba de uso hospitalar para infusão contínua. Esse equipamento é utilizado rotineiramente em hospitais humanos - nas unidades pediátricas para controle do volume de soluções ministradas via intravenosa. Por meio de um equipo próprio, o controle da vazão torna-se altamente preciso com a utilização da bomba de infusão, pois o volume é controlado eletronicamente.

A aplicação dessa bomba para fins de aspiração folicular foi possível graças ao modo inverso de utilização do equipo de infusão. A extremidade a ser ligada ao frasco de infusão foi acoplada no tubo de $50 \mathrm{ml}$ destinado ao armazenamento do fluido aspirado, enquanto a extremidade do equipo destinada ao cateter do paciente foi mantida livre (Figuras 2 a 4).

Dessa maneira, foi possível a manutenção constante de pressão negativa capaz de promover a aspiração dos folículos. Como aspectos favoráveis da bomba de infusão, além de seu menor custo, ressaltase seu reduzido tamanho, controle eletrônico da vazão, ausência de ruído e bateria com capacidade para seis

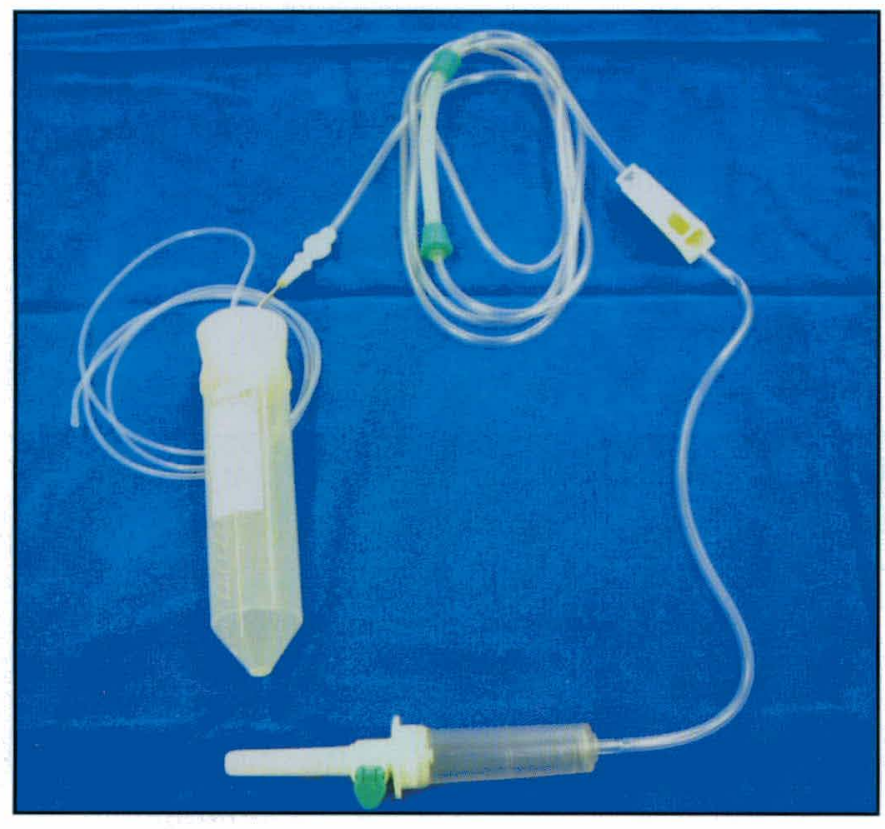

Figura 2 - Foto ilustrando a conexão do equipo de infusão na tampa do tubo cônico para acondicionamento do fluido a ser obtido pela punção folicular

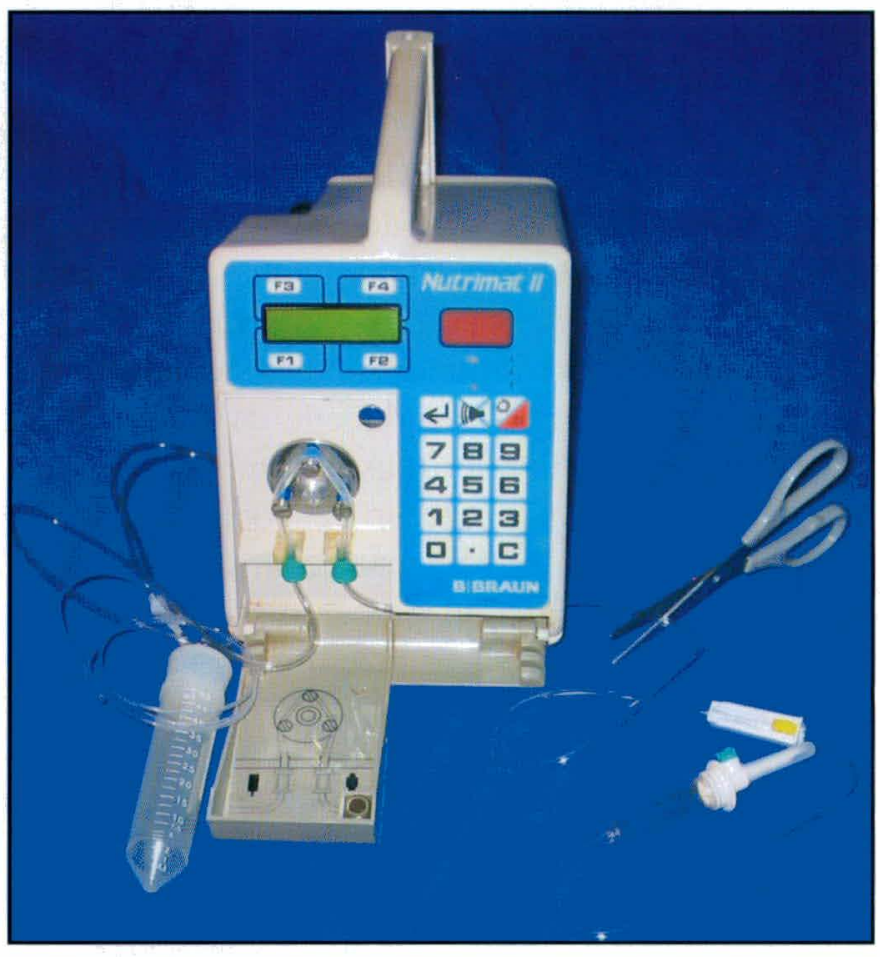

Figura 3 - Foto demonstrativa da montagem do equipo na bomba de infusão. A tesoura ilustra o segmento do equipo a ser cortado, uma vez que apenas a outra extremidade é utilizada

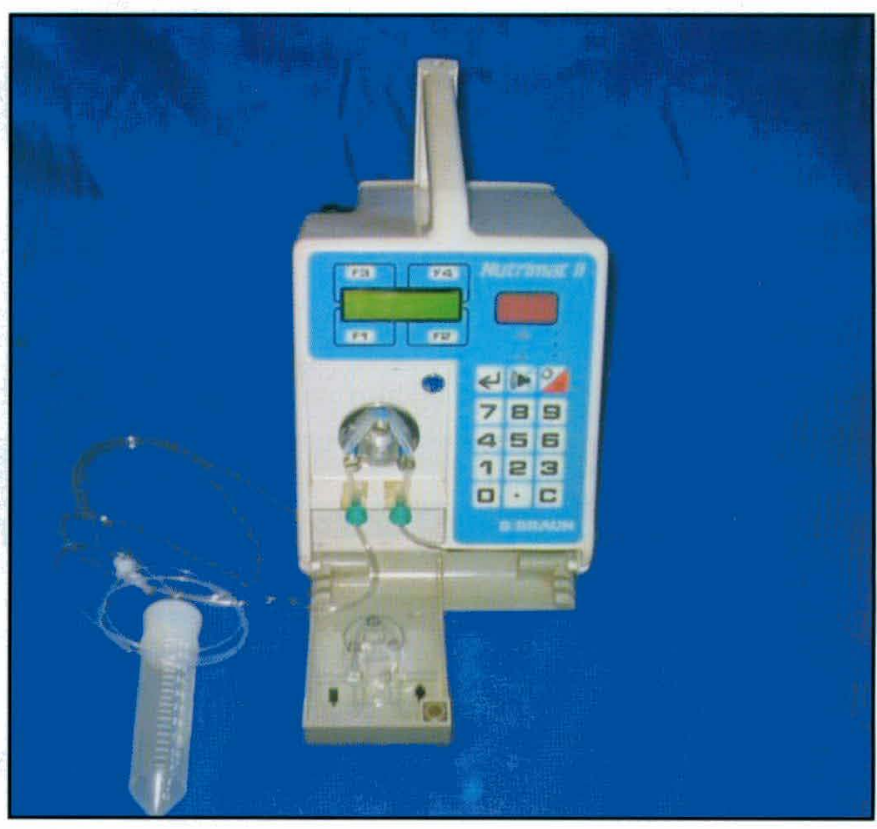

Figura 4 - Foto com o conjunto - bomba e tubo de coleta - pronto para ser utilizado na obtenção in vivo de oócitos na espécie bovina 
horas de trabalho. Destaca-se também a facilidade de aquisição de equipamentos usados, a serem adquiridos em unidades neonatais ou de hemodiálise em hospitais humanos. Como aspecto desfavorável, citase a demora na formação do vácuo no início do procedimento, aspecto corrigido com a manutenção do equipamento em operação ininterrupta, desde o primeiro até o último animal a ser puncionado. Outro ponto negativo seria a necessidade de aquisição de um equipo específico de infusão. Embora este seja de baixo custo, normalmente só é encontrado em lojas de material hospitalar. Sugere-se a utilização de um equipo por dia de trabalho, para minimizar o risco de microfissuras no equipo, capazes de causar perda de eficiência do vácuo.

Rubin et al. ${ }^{27}$ (2004) obtiveram 704 oócitos em 28 procedimentos de aspiração folicular utilizando a bomba de infusão contínua em vacas Nelore, com média de 25.14 oócitos viáveis por sessão de aspiração. Esses resultados foram similares aos citados na literatura com a utilização de equipamentos delineados especificamente para aspiração folicular. Sugerem, portanto, a possibilidade da utilização da bomba de infusão de forma satisfatória, viabilizando a obtenção de oócitos in vivo, com níveis adequados de eficiência.

A tabela 1 mostra o número de oócitos recuperados, bem como a média de oócitos viáveis por sessão de OPU, pela utilização de dois tipos de bombas de vácuo.

\section{Considerações Finais}

A opção pelo tipo de bomba de vácuo deve levar em consideração diversos fatores, como a disponibilidade de recursos a serem investidos, e qual a demanda da aspiração folicular dentre as demais atividades do profissional. Modelos destinados especificamente para a aspiração folicular permitem mais eficiência, mas o seu preço os torna inacessíveis aos profissionais que não se dedicam exclusivamente à aspiração folicular. Dentre os modelos alternativos de menor custo, a bomba de infusão contínua apresenta vantagens importantes, como controle eletrônico da vazão, bateria para até seis horas de trabalho, baixíssimo nível de ruído e eficiência satisfatória, quando comparada às bombas específicas para punção. Portanto, a bomba de infusão contínua pode ser uma alternativa viável para obtenção in vivo de oócitos bovinos.

\section{Agradecimentos}

Ao Sr. Renato Castanho Francisco da Embryomas ${ }^{\circledast}$ pela proposta de utilização da bomba de infusão contínua.

Tabela 1 - Número médio de oócitos recuperados pela OPU com a utilização de duas bombas de vácuo diferentes

\begin{tabular}{ccccc}
\hline Autor & $\begin{array}{c}\text { No de } \\
\text { procedimentos }\end{array}$ & $\begin{array}{c}\text { No de oócitos } \\
\text { recuperados }\end{array}$ & $\begin{array}{c}\text { Média de oócitos } \\
\text { viáveis/aspiração }\end{array}$ & $\begin{array}{c}\text { Bomba geradora } \\
\text { de vácuo }\end{array}$ \\
\hline $\begin{array}{c}\text { Nonato Jr. et al. }{ }^{28} \\
(2004)\end{array}$ & 85 & 2.270 & 26.70 & Handle Cook $^{\circledR}$ \\
$\begin{array}{c}\text { Rubin et al. }{ }^{17} \\
(2004)\end{array}$ & 28 & 704 & 25.14 & $\begin{array}{c}\text { Bomba de infusão } \\
\text { contínua }\end{array}$ \\
\hline
\end{tabular}




\section{Abstract}

To disclose an alternative equipment for vacuum generation, which allows for oocyte recovery in quality and quantity compatible with specific vacuum pumps. A continuous infusion pump was employed and aspiration was possible due to the use of the infusion fluid line in the reverse way. The end destined for the infusion bottle was connected to the follicle aspiration tube, while the end destined for the patient's catheter was kept free. The results were similar to those obtained with specific follicle aspiration equipments. The continuous infusion pump can represent a viable alternative for recovery of bovine oocytes in vivo.

Keywords: Ovary follicle. Oocytes. Follicle aspiration. Vacuum. Ultrasonography. Bovine.

\section{Resumen}

Divulgar un equipo alternativo para la generación del vacuo, capaz de permitir la recuperación de oocitos en la cantidad y calidad compatible con las bombas hechas específicamente para ello. Se usó una bomba de infusión continua, cuya aplicabilidad para fines de aspiración fue posible gracias a la manera inversa de uso del equipo de infusión. La extremidad destinada al frasco de infusión fue acoplada en el tubo de aspiración folicular, mientras la extremidad del equipo destinada al catéter del paciente fue mantenida libre. Se obtuvieron resultados similares a los encontrados en equipos designados específicamente para aspiración folicular. La bomba de infusión continua puede ser una alternativa viable para la obtención in vivo de oocitos bovinos.

Palabras- clave: Folículo ovárico. Oocitos. Aspiración folicular. Vacuo. Ultrasonografía. Bovinos.

\section{Referências}

1. ERICKSON, B. H. Development and senescence of the postnatal bovine ovary. Journal of Animal Science, v. 25, p. 800-805, 1966.

2. EPPIG, J. J.; O'BRIEN, M. J. Developmental in vitro of mouse oocytes from primordial follicles. Biology of Reproduction, v. 54, p. 197-207, 1996.

3. BOLS, P. E. J. et al. A comparison of a mechanical sector and a linear array transducer for ultrasoundguided transvaginal oocyte retrieval (OPU) in the cow. Theriogenology, v. 62, p. 906-914, 2004.

4. SENEDA, M. M.; BLASCHI, W. Ovum pick up em bovinos: considerações técnicas. In: SIMPÓSIO INTERNACIONAL DE REPRODUÇÃO ANIMAL APLICADA, 1., 2004, Londrina, PR. Anais... Londrina, 2004. p. 231-237.

5. BOLS, P. E. J. Transvaginal ovum pick-up in the cow: technical and biological modifications. Ghent, 1997. 227 p. Thesis (PhD)- Faculty of Veterinary Medicine, University of Ghent, Belgium, 1997. 
6. HASHIMOTO, S. et al. Ultrasound-guided follicle aspiration: effect of the frequency of a linear transvaginal probe on the collection of bovine oocytes. Theriogenology, v. 52, p. 131-138, 1999.

7. CALLESEN, H. et al. Ultrasonically guided aspiration of bovine follicular oocytes. Theriogenology, v. 27, p. 217, 1987. (Abstract).

8. HASLER, J. F. et al. Production, freezing and transfer of bovine IVF embryos and subsequent calving results. Theriogenology, v. 43, p. 141-152, 1995.

9. MANIK, R. S. et al. Collection of oocytes through transvaginal ultrasound-guided aspiration of follicles in an Indian breed of cattle. Animal Reproduction Science, v. 76, p. 155-161, 2003.

10. ROOVER, R. et al. Ovum pick up and in vitro embryo production in cows superstimulated with an individually adapted superstimulation protocol. Animal Reproduction Science, v. 86, p. 13-25, 2005.

11. BUNGARTZ, L. et al. Collection of oocytes from cattle via follicular aspiration aided by ultrasound with or without gonadotropin pretreatment and in different reproductive stages. Theriogenology, v. 43, p. 667 $675,1995$.

12. HENDRIKSEN, P. J. M. et al. Effect of different stages of the follicular wave on in vitro developmental competence of bovine oocytes. Theriogenology, v. 61, p. 909-902, 2004.

13. VAN DER SCHANS, A. et al. Ultrasound-guided transvaginal collection of oocytes in the cow. Theriogenology, v. 35, p. 288, 1991. (Abstract).

14. SAUVÉ, R. Ultrasound guided follicular aspiration and in vitro fertilization. Arquivos da Faculdade de Veterinária UFRGS, v. 26, p. 141-155, 1998.

15. AERTS, J. M. J. et al. The use of an endovaginal ultrasound micro-convex array transducer adapted for tranvaginal oocyte retrieval in the cow. INTERNATIONAL CONGRESS ON ANIMAL REPRODUCTION, 15., 2004, Porto Seguro, Brasil. Abstracts... Belo Horizonte: Colégio Brasileiro de Reprodução Animal, 2004. v. 2, p. 435.
16. LOONEY, C. R. et al. Commercial aspects of oocyte retrieval and in vitro fertilization (IVF) for embryo production in problem cows. Theriogenology, v. 41, p. 67-72, 1994.

17. MEINTJES, M. et al. Transvaginal aspiration of oocytes from hormone-treated pregnant beef for in vitro fertilization. Journal of Animal Science, v. 73 , p. $967-974,1995$

18. BOLS, P. E. J. et al. Effects of aspiration vacum and needle diameter on cumulus oocyte complex morphology and developmental capacity of bovine oocytes. Theriogenology, v. 45, p. 1001-1014, 1996.

19. BOLS, P. E. J. et al. Effects of long-term treatment with bovine somatotropin on follicular dynamics and subsequent oocyte and blastocyst yield in an OPUIVF program. Theriogenology, v. 49, p. 983-995, 1998.

20. CARLIN, S. K. et al. Effects of ultrasound-guided transvaginal follicular aspiration on oocyte recovery and hormonal profiles before and after GnRH treatment. Theriogenology, v. 51, p. 1489-1503, 1999.

21. SENEDA, M. M. et al. Efficacy of linear and convex transducers for ultrasound-guided transvaginal follicle aspiration. Theriogenology, v. 59, p. 1435-1440, 2003

22. PIETERSE, M. C. et al. Aspiration of bovine oocytes during transvaginal ultrasound scanning of the ovaries. Theriogenology, v. 30, p. 751-762, 1988.

23. BOLS, P. E. J. et al. Effects of needle tip bevel and aspiration procedure on the morphology and developmental capacity bovine compact cumulus oocyte complexes. Theriogenology, v. 47, p. 1221-1236, 1997.

24. BOLS, P. E. J. et al. Transvaginal oocyte pick-up in infertile Belgium blue donor cows: preliminary results. Theriogenology, v. 45, p. 359, 1996. (Abstract).

25. VOS, P. L. A. M. et al. Evaluation of transvaginal ultrasound-guided follicle puncture to collect oocytes and follicular fluids at consecutive times relative to the preovulatory LH surge in eCG/PG treated cows. Theriogenology, v. 41, p. 829-840, 1994. 
26. RICK, G. et al. Long-term ultrasound guided ovum pick-up in heifers from 6 to 15 months of age. Theriogenology, v. 45, p. 356, 1996.

27. RUBIN, K. C. P. et al. Avaliação de uma bomba de infusão contínua como geradora de vácuo para obtenção in vivo de oócitos bovinos.

Acta
Scientiae Veterinariae, v. 32, p. 120, 2004. (Abstract).

28. NONATO JÚNIOR, I. et al. Produção de embriões em vacas Nelore com a utilização associada de FIV e TE. Acta Scientiae Veterinariae, v. 32, p. 95, 2004. (Abstract). 